\title{
Giant Magnetoresistance and Shubnikov-de Haas Effect in LuSb
}

\author{
M. Kleinert, O. Pavlosiuk, P. Swatek, D. Kaczorowski, P. Wiśniewski* \\ Institute of Low Temperature and Structure Research, Polish Academy of Sciences, \\ P.O. Box 1410, 50-950 Wrocław, Poland
}

\begin{abstract}
Single-crystals of LuSb were investigated by means of electrical resistivity and magnetoresistance measurements. The compound was found to exhibit giant magnetoresistance exceeding $3000 \%$, low-temperature resistivity plateau, and Shubnikov-de Haas oscillations. It was characterized as a semimetal with nearly balanced contributions of electron and hole carriers to the magnetotransport properties. The experimental findings, supported by the results of electronic structure calculations, proved that the magnetotransport in LuSb can be described in the scope of 3D multi-band Fermi surface model without topologically non-trivial electronic states.
\end{abstract}

DOI: 10.12693/APhysPolA.133.538

PACS/topics: 71.20.Eh, 71.55.Ak, 72.15.Eb

\section{Introduction}

Lanthanum monopnictides have recently been elaborated as materials with non-trivial topology of their electronic structures $[1,2]$. In the case of Lu-based pnictides, there are several theoretical reports describing structural and physical properties [3, 4], however hitherto experimental studies were focused only on structural phase transitions at high pressures $[5,6]$. In the present work, we report giant magnetoresistance and Shubnikovde Haas oscillations in LuSb. The experimental work is supplemented by our calculations of electronic band structure of this compound.

\section{Experimental details}

Single crystals of LuSb were grown in a two-step process. First, polycrystalline sample was synthesized by arc-melting under argon atmosphere. Stoichiometric amounts of high purity $(>3 N)$ constituents were used.

The second step was growing LuSb crystals by flux method. The polycrystalline ingot was ground, mixed with Sn pieces in molar ratio 1:20, loaded into alumina crucible and sealed in evacuated quartz tube. The sample was heated to $1160^{\circ} \mathrm{C}$ at $21^{\circ} \mathrm{C} / \mathrm{h}$ rate, and then held at this temperature for 20 hours. Subsequently, it was slowly cooled to $800^{\circ} \mathrm{C}$ at a rate of $1{ }^{\circ} \mathrm{C} / \mathrm{h}$.

Composition of the so-obtained single crystals was checked by energy-dispersive X-ray spectroscopy, and their crystal structure was verified by X-ray diffraction with $\mathrm{Cu}-\mathrm{K}_{\alpha}$ radiation. The cubic lattice parameter of $6.0608 \AA$ was determined. The single-crystals were orientated by standard Laue diffraction technique (see Fig. 1). Electrical transport measurements were carried out in the

\footnotetext{
* corresponding author; e-mail: P.Wisniewski@int.pan.wroc.pl
}

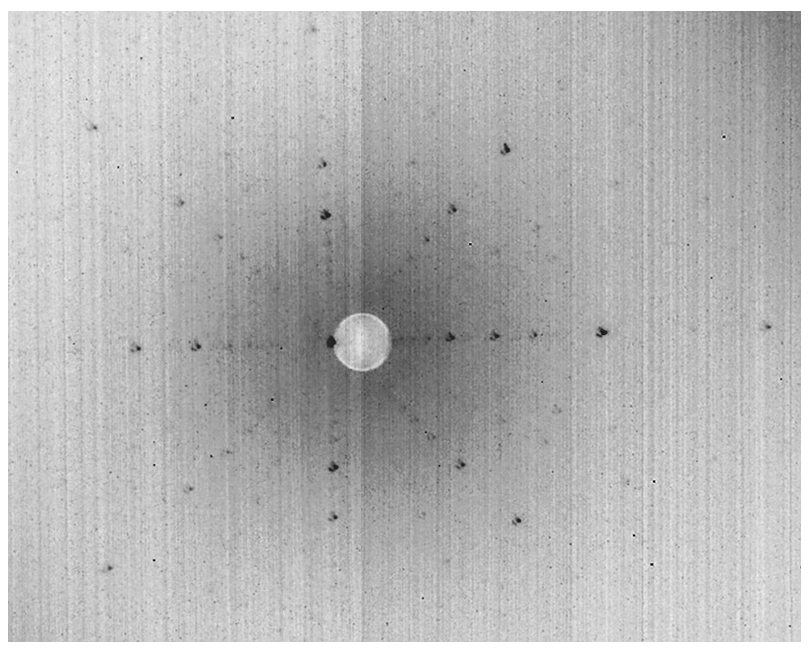

Fig. 1. Laue pattern of LuSb. Central spot refers to (001) plane.

temperature range $0.4-300 \mathrm{~K}$ in magnetic fields up to $9 \mathrm{~T}$ using a Quantum Design PPMS platform.

\section{Results and discussion}

\subsection{Electrical resistivity}

The temperature variation of the electrical resistivity, $\rho$, of single-crystalline LuSb is shown in Fig. 2. Similarly to YSb [7] and LaSb [8], the compound exhibits semimetallic-like behaviour, with a characteristic resistivity plateau, observed below about $10 \mathrm{~K}$ in a magnetic field applied perpendicular to the electrical current.

The transverse magnetoresistance, defined as $M R \equiv$ $\left[\rho(B)-\left.\rho\right|_{B=0}\right] /\left.\rho\right|_{B=0}$, is plotted in Fig. 3 versus magnetic field. The MR isotherms exhibit a usual parabolic shape. Below $15 \mathrm{~K}, \mathrm{MR}$ reaches as large value as $3000 \%$ in a field of $9 \mathrm{~T}$. With increasing temperature, MR measured in this field rapidly decreases; it is equal to $642 \%$ at $50 \mathrm{~K}$, and only $8 \%$ at $300 \mathrm{~K}$. 


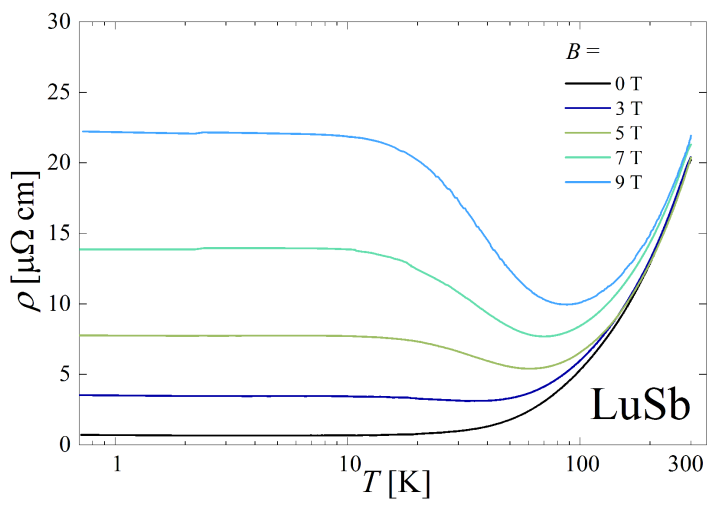

Fig. 2. Electrical resistivity of LuSb as a function of temperature, measured in different magnetic fields.

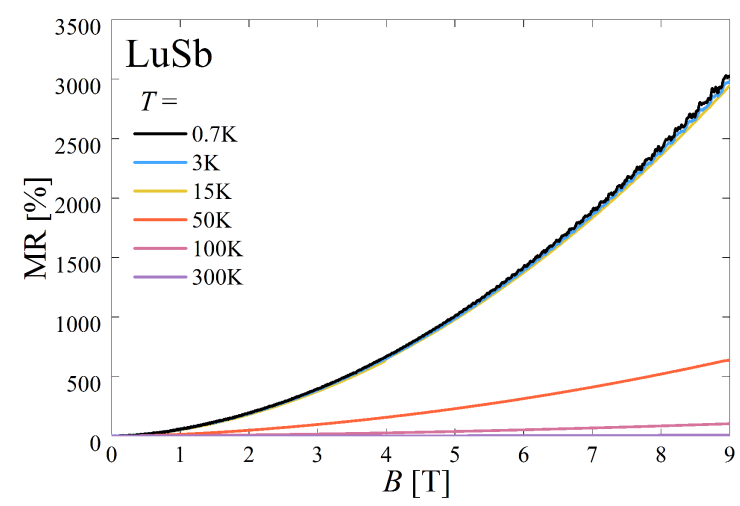

Fig. 3. Magnetic field dependence of the magnetoresistance of LuSb measured at different temperatures.

\subsection{Shubnikov-de Haas (SdH) oscillations}

Alike for the lanthanum monopnictides [9], the electrical conductivity in LuSb exhibits distinct quantum oscillations in relatively weak magnetic fields. The $\mathrm{SdH}$ oscillations were well resolved even at $15 \mathrm{~K}$, which indicates very good quality of the single crystals studied. Figure 4 shows the oscillatory component $\Delta \rho$ vs $B^{-1}$, obtained from the $\rho(B)$ isotherms by subtracting background. Fast Fourier transform (FFT) analysis yielded six frequencies listed in Table I.

The data were evaluated in terms of the LifshitzKosevich (L-K) expression:

$$
\Delta \rho \sim \sum_{i=\alpha, \beta, \delta, \gamma} \cos \left[2 \pi\left(\frac{f_{i}}{B}-\varphi_{i}-\frac{1}{8}\right)\right],
$$

where $f_{i}$ are the $\alpha, \beta, 2 \alpha, \alpha_{1}, 2 \beta$ and $\delta$ frequencies obtained from the FFT analysis and $\varphi_{i}$ represent the corresponding phase shifts. The so-obtained values of $\varphi_{i}$ are collected in Table I. The knowledge of phase shift, which directly corresponds to Berry phase, allows to conclude about the carriers nature. For Dirac fermions, $\varphi$ equals to 0.5 , whereas for Schrödinger fermions it equals to 0 . The obtained values of $\varphi_{i}$ are very close to 0 , thus the observed SdH oscillations do not reveal Dirac fermions in LuSb.

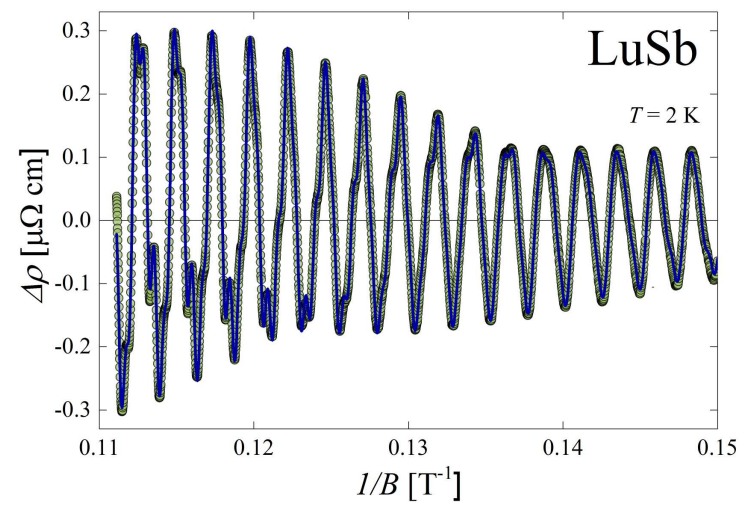

Fig. 4. Oscillatory component of the electrical resistivity of LuSb at $2 \mathrm{~K}$ plotted versus $B^{-1}$. Blue solid line represents the fit with L-K formula.

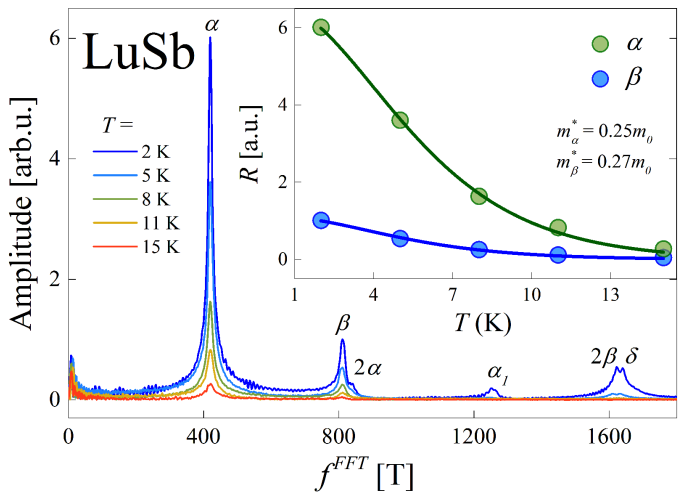

Fig. 5. Amplitudes of the $\mathrm{SdH}$ oscillations in LuSb at different temperatures, obtained from the FFT analysis. Inset: amplitudes of the FFT peaks fitted with Eq. 2.

Figure 5 displays the amplitudes of the SdH oscillations observed at different temperatures. As shown in the inset to this figure, the temperature dependence of the FFT peaks can be well approximated by the formula

$$
R(T)=\left.\left(\left(\frac{\lambda m_{i}^{*} T}{B}\right) / \sinh \left(\frac{p \lambda m_{i}^{*} T}{B}\right)\right)\right|_{B=9 \mathrm{~T}},
$$

where $m_{i}^{*}$ are the effective masses of charge carriers and the constant $\lambda=2 \pi^{2} \hbar \approx 14.7 \mathrm{~T} / \mathrm{K}$. This way, the values of $m_{\alpha}^{*}=0.25 m_{e}$ and $m_{\beta}^{*}=0.27 m_{e}$ were determined. These effective masses are relatively small but comparable to those observed for other rare earth monopnictides $[7,8]$.

TABLE I

Parameters obtained from SdH oscillations analysis.

\begin{tabular}{c|c|c|c|c|c|c}
\hline \hline$i=$ & $\alpha$ & $\beta$ & $2 \alpha$ & $\alpha_{1}$ & $2 \beta$ & $\delta$ \\
\hline$f_{i}^{F T}[\mathrm{~T}]$ & 420 & 811 & 840 & 1252 & 1623 & 1640 \\
$\varphi_{i}$ & 0.15 & 0.08 & 0.34 & 0.13 & 0.06 & 0.14
\end{tabular}

\subsection{Electronic structure calculations}

The calculations were performed using the all-electron general potential linearized augmented plane-wave 
(LAPW) method, employing the WIEN2k code [10]. For the Fermi surface (see Figure 6) the irreducible Brillouin zone was sampled by $20225 \boldsymbol{k}$ points to ensure accurate determination of the Fermi level [11]. We got a very good agreement between the results of calculations and experimental ones. SdH oscillations, which we observed, contain frequencies originating from three Fermi pockets $\alpha, \beta$ and $\delta$. Only the smallest $\gamma$ Fermi sheet was not discernible in the oscillations.

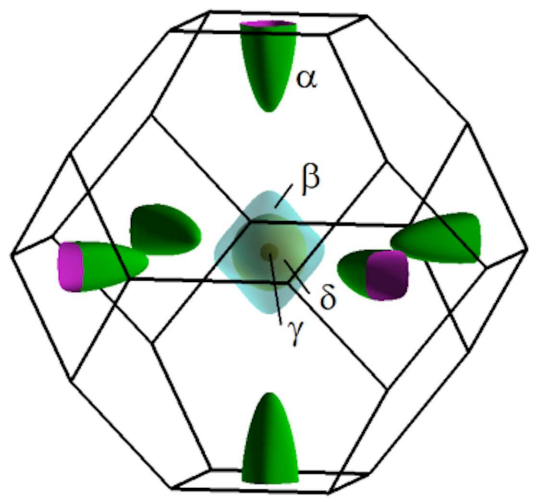

Fig. 6. Fermi surfaces in LuSb. Labels $\alpha, \beta, \gamma$ and $\delta$ correspond to the $\phi_{i}$ frequencies in the FFT analysis.

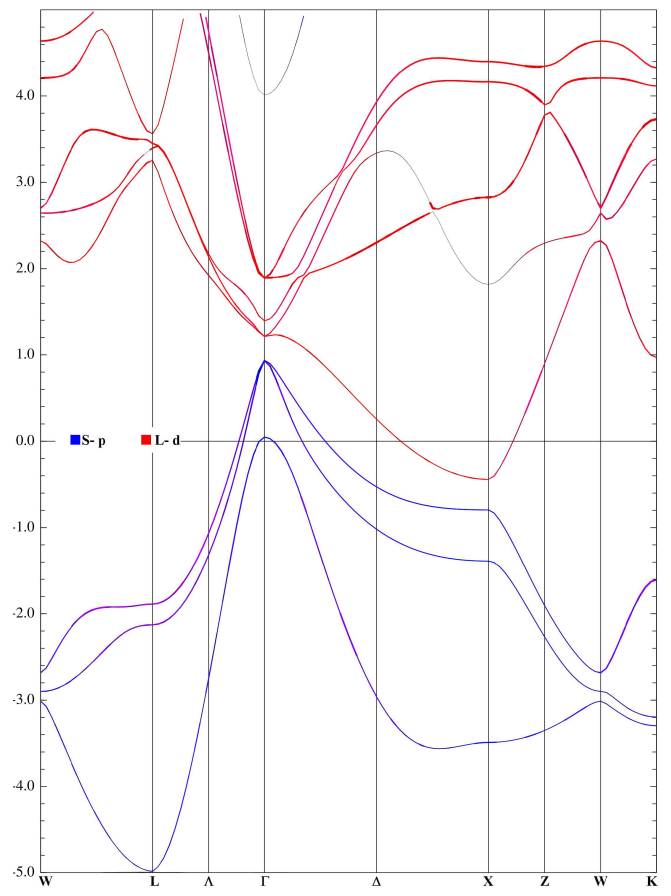

Fig. 7. Calculated electronic band structure of LuSb. Red and blue lines represent the contribution due to $\mathrm{Sb}-$ $p$ bands and Lu- $d$ bands, respectively. $E=0$ denotes the Femi level.
The electronic band structure of LuSb is shown in Fig. 7. There are three hole pockets nested at the centre of Brillouin zone $\Gamma$ and electron pocket near the highsymmetry $X$ point. Remarkably, band inversion occurs near $X$ point, caused by mixed $p-d$ character of bands. This may lead to the formation of Dirac cones.

\section{Conclusions}

Our experimental study of the Shubnikov-de Haas oscillations in LuSb, complemented with the ab initio electronic band structure calculations indicated that its Fermi surface consists of one electron and three hole pockets. Comparison of volumes of these pockets reveal that LuSb is a semimetal close to compensation. The band inversion near the $X$ point of the Brillouin zone may give rise to topologically non-trivial electronic states, however the experimental magnetotransport data did not confirm their presence. Spectroscopic investigations (e.g. ARPES, STM) are needed to resolve the actual topological character of this material.

\section{Acknowledgments}

This work was supported by the National Science Centre (Poland); grant no. 2015/18/A/ST3/00057.

\section{References}

[1] M. Zeng et al., arXiv:1504.03492 (2015).

[2] J. Nayak et al., Nat. Commun. 8, 13942 (2017).

[3] G. Pagare et al., Comput. Mater. Sci. 50, 538 (2010).

[4] G. Pagare et al., J. Phys. Conf. Ser. 215, 012114 (2010).

[5] R.J. Birgeneau et al., Phys. Rev. B 8, 5345 (1973).

[6] I. Shirotani, et al., Phys. Rev. B 64, 132101 (2001).

[7] O. Pavlosiuk, P. Swatek, P. Wiśniewski, Sci. Rep. 6, 38691 (2016).

[8] F.F. Tafti, et al., Nat. Phys. 12, 272 (2016).

[9] A. Hasegawa, J. Phys. Soc. Jpn. 54, 677 (1985).

[10] P. Blaha et al., WIEN2K, An Augmented Plane Wave + Local Orbitals Program for Calculating Crystal Properties, Techn. Universität Wien, Austria 2001.

[11] A. Kokalj, Comp. Mater. Sci. 28, 155 (2003); code available from www.xcrysden.org/. 